\title{
Intermolecular Forces Dictate Vibrational Energy Transfer in Plasmonic-Molecule Systems
}

\section{Supporting Information}

Ziwei Yu and Renee R. Frontiera*

Department of Chemistry, University of Minnesota, Minneapolis, MN 55455

*to whom correspondence should be addressed: rrf@umn.edu 


\section{Method}

Sample Preparation and Characterization

We synthesized citrate-capped gold nanoparticles following the Frens method, ${ }^{1}$ where 5.0 $\mathrm{mg}$ of gold (III) chloride dihydrate was dissolved in $50 \mathrm{~mL}$ of Milli-Q water and heated until boiling while stirred. To make gold nanoparticles with a reported diameter of $97.5 \mathrm{~nm}$, we added $0.21 \mathrm{~mL}$ of $1 \%$ sodium citrate solution to the boiling gold (III) chloride dihydrate solution and heated the mixed solution until no further color change was observed. To remove the free citrate and sodium ions, we centrifuged the as-synthesized gold colloid solutions for $40 \mathrm{~min}$ at $3000 \mathrm{rpm}$ and took off the supernatant. We diluted the remained concentrated gold nanoparticles into the original volume with milli Q water and left it overnight. We centrifuged the diluted solution for $30 \mathrm{~min}$ at $2500 \mathrm{rpm}$ and removed the supernatant to make a 100x concentrated gold colloid solution. To fabricate SERS substrates, we drop casted $250 \mu 1$ of the 100x concentrated gold colloid solution onto a 22mm x 22mm piece of filter paper (Fisherbrand, Qualitative Filter Paper, Cellulose, Grade P8) and let it dry at ambient condition. We made $300 \mu \mathrm{M}$ thiol solutions in a 50:50 mixture of water and methanol and drop casted $250 \mu \mathrm{l}$ of the solution on each SERS substrate. We let the substrates dry at ambient condition to achieve full adsorption of aromatic thiols on the gold surface. We obtained all chemicals from Sigma-Aldrich and used without further purification.

We measured the extinction spectra of the SERS substrates with a Shimadzu UV-2600 UVVis spectrometer with an integrating sphere at an 8-degree reflection geometry. In Figure S1, we show the extinction spectra of the SERS substrates with different aromatic thiols as the analyte. The plasmonic characteristics of each substrate are nearly identical, featuring a high degree of sample consistency. Along with the peak around $535 \mathrm{~nm}$ representing the monomer mode of the gold nanoparticles, the extinction spectra show a broad band at the red side, which implies the 
heterogenous packing of the aggregated nanoparticles.

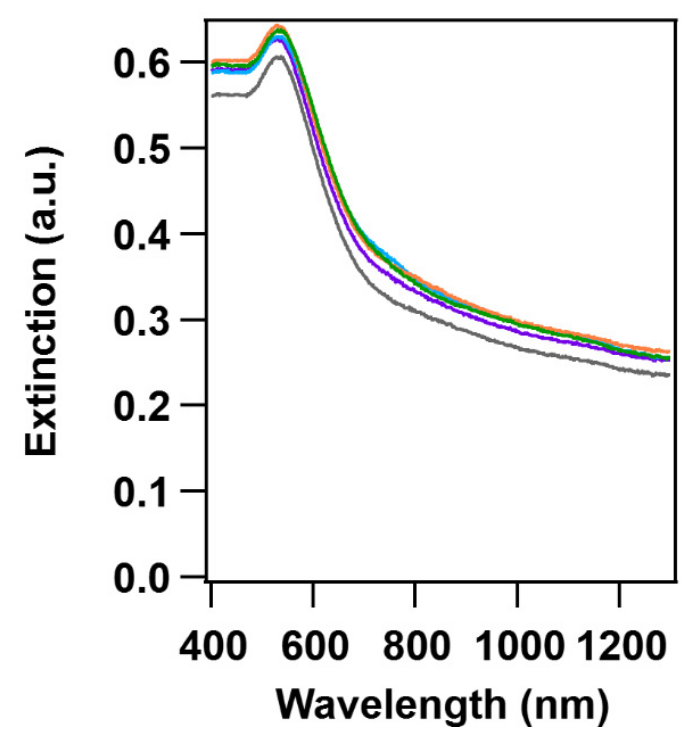

Figure S1. Extinction spectra of the SERS substrates with 4-methylbenzenethiol (MTP, purple), 4-chlorobenzenethiol (CTP, orange), 4-nitrobenzenethiol (NBT, green), biphenyl-4-thiol (PTP, blue) and benzenethiol (BT, gray). Spectra are overlapped because the substrates are nearly identical.

We characterized the SERS substrates with a JOEL 6500 scanning electron micrograph (SEM). The SEM images (Figure S2) of the SERS substrates reveal the packing of gold nanoparticles into clusters on the microfibers of the filter paper, agreeing well with the extinction spectra. The gold nanoparticles have a wide size distribution, ranging from $67 \mathrm{~nm}$ to $100 \mathrm{~nm}$. To account for sample heterogeneity across the substrate, we did the same ultrafast measurements on three different locations on each substrate and report them as distinct results. 


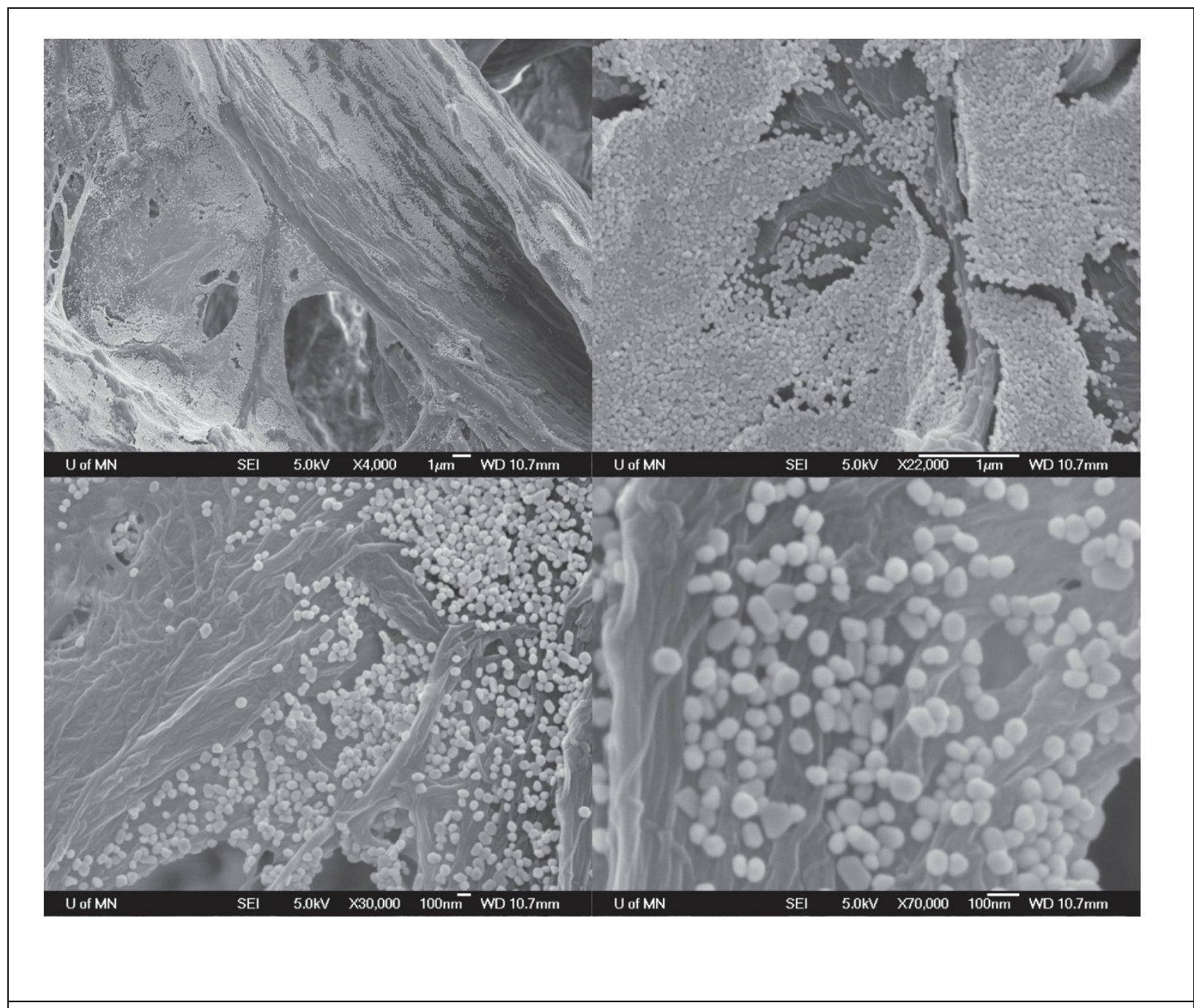

Figure S2. SEM images of the SERS substrate.

\section{Instrument Set-up}

We performed ultrafast SERS measurements on a home-built instrument as described in previous literature. ${ }^{2}$ In brief, a fundamental beam of $<250$ fs laser pulses centered at $1035 \mathrm{~nm}$ with the repetition rate of $2.04 \mathrm{MHz}$ was generated from a diode-pumped Yb-fiber-amplified laser (ClarkMXR Impulse). We split the fundamental beam by a 50:50 beam splitter and sent half of the fundamental beam to a spectral filter to generate the narrowband picosecond probe beam, where the beam was dispersed by a transmission grating and then focused to a slit by a cylindrical lens. 
We used the rest of the fundamental beam directly as the pump beam. We used a motorized delay stage (Newport XMS500) to change the path length of the pump beam, creating various time delay between the pump and probe. When the pump arrives at the sample after and before the probe, they are referred to as negative and positive timepoints respectively. When they arrive at the same time, it is called time zero. We collected the scattered photons in a 90-degree reflection geometry. We used a $1000 \mathrm{~nm}$ short pass filter for anti-Stokes scattering and a $1064 \mathrm{~nm}$ long pass filter for Stokes scattering. We used a 1024-pixel InGaAs array (Princeton Instruments PYLON-IR 1.7) with a $600 \mathrm{gr} / \mathrm{mm}$ grating blazed at $750 \mathrm{~nm}$ (Princeton Instruments, 2300i) for detection. The time resolution of the setup is 2.0 ps as measured with optical Kerr effect in toluene. The spot size is $6.8 \times 10^{-5} \mathrm{~cm}^{2}$ for the probe beam and $4 \times 10^{-4} \mathrm{~cm}^{2}$ for the pump beam as calculated with the following equation:

$$
\text { Spot radius }=2 \sqrt{\frac{-2\left(\frac{d}{2}\right)^{2}}{\ln \left(1-\frac{p_{a}}{p_{b}}\right)}}
$$

where $\mathrm{d}$ is the diameter of the pinhole, $p_{a}$ is the power of the laser beam measured after the pinhole and $p_{b}$ is the power measured before the pinhole.

Throughout the experiment, the average power of the picosecond and femtosecond pulses are both $20 \mathrm{~mW}$ at the sample, which corresponds to a power flux of $300 \mathrm{~W} / \mathrm{cm}^{2}$ for the picosecond pulse and $50 \mathrm{~W} / \mathrm{cm}^{2}$ for the femtosecond pulse. When the sample stays stationary, the sample gets damaged and the enhancement diminishes within minutes. To compensate for this issue, we rotated the SERS substrates at a speed of 7200 rpm with a home-built spinning stage based on a computer hard drive, which mitigates the sample damage from the high-energy pulsed laser. Figure S3a shows the magnitude of the Stokes scattering with the sample being illuminated by the $20 \mathrm{~mW}$ picosecond pulse for $30 \mathrm{~min}$ and by the $20 \mathrm{~mW}$ picosecond pulse overlapped with the $20 \mathrm{~mW}$ 
femtosecond pulse for another 30 min when the sample was spinning. The Stokes scattering intensity from the SERS substrate with $20 \mathrm{~mW}$ picosecond laser illumination experienced less than 5\% loss over 30 min with the spinning. The signal magnitude experienced a sharper decrease with the illumination of $20 \mathrm{~mW}$ femtosecond laser, which is due to the much higher peak power density of the femtosecond laser. With further illumination, the signal still reached a relative stable stage even with the femtosecond laser illumination. Therefore, for all the samples, we illuminated the substrates until the Stokes intensity is relatively stable and took two sets of transient data on the same location with an additional one-hour illumination in between. To be consistent, we controlled the illumination time to be the same for all samples. As shown in Figure S3b, the kinetic traces extracted from the two sets of transient data taken at the same location exhibit little difference, which proves that the substrates reached a stabilized state when we took the measurement. 


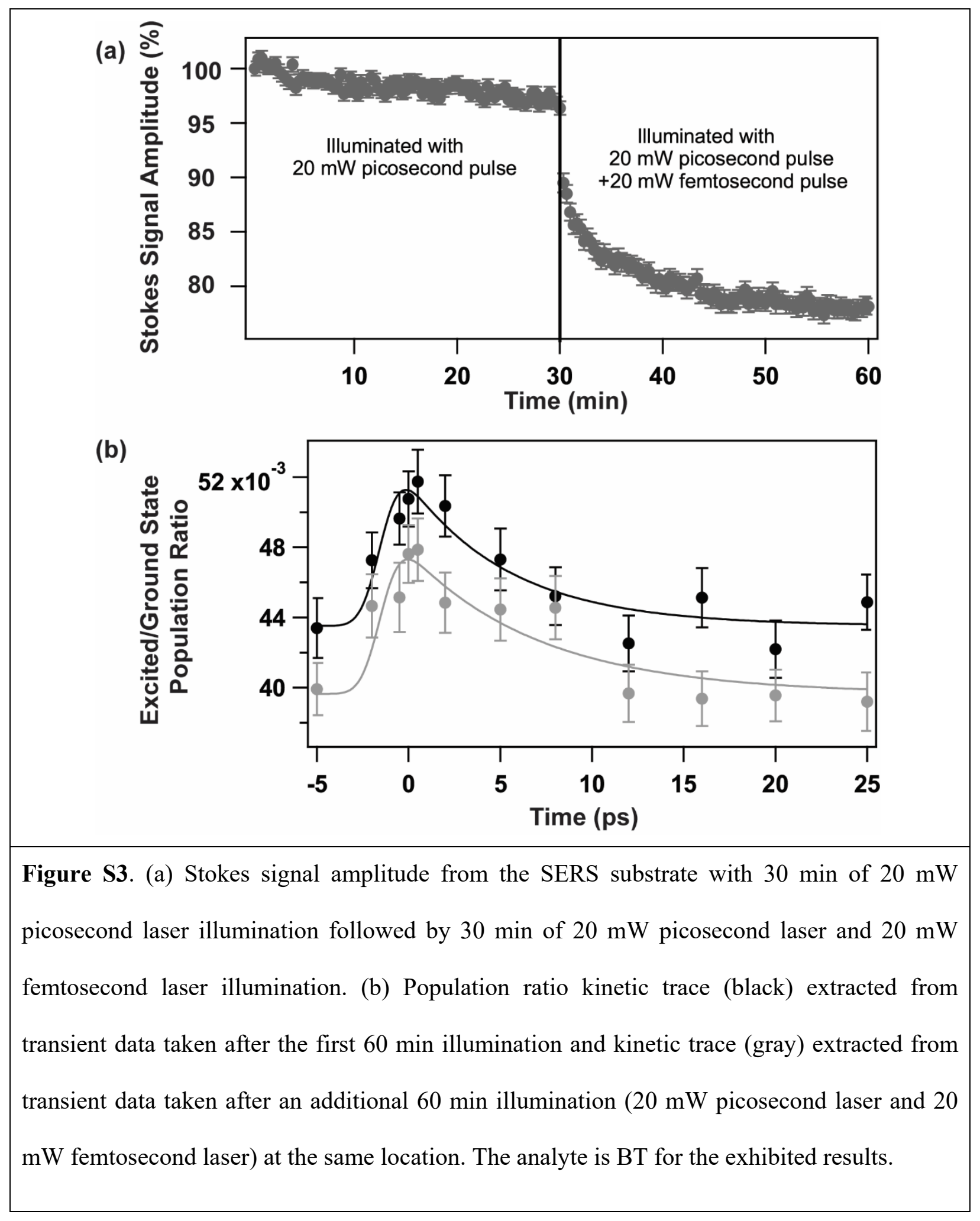




\section{Data Collection and Processing}

We took the ultrafast SERS measurements with a home-written Labview program. Each Stokes scan consists of Stokes spectra taken at different time delays, as does the anti-Stokes scan. Each complete set of transient data has one Stokes scan and two anti-Stokes scans, with the Stokes scan taken between the two anti-Stokes scans. We averaged the anti-Stokes spectra of corresponding time delays from the two scans together to eliminate the bias introduced by sample degradation over the period of measurement. At each timepoint, we used a pump-only spectrum of the same acquisition time to subtract out the pump-induced broad background. The acquisition time is 30s for anti-Stokes spectra and 20s for Stokes spectra. We normalized the intensity of each spectrum with the acquisition time. For each sample, we took the measurement at three different locations. At each location, we first illuminated the sample for $30 \mathrm{~min}$ with a $20 \mathrm{~mW}$ picosecond laser, and then $60 \mathrm{~min}$ with both a $20 \mathrm{~mW}$ picosecond and a $20 \mathrm{~mW}$ femtosecond laser overlapped. This step is to stabilize the substrate as shown in Figure S3a and minimize the discrepancy introduced by sample degradation during the measurement. After the illumination, we took the first set of transient data. Then we illuminated the substrate for another $60 \mathrm{~min}$ with the $20 \mathrm{~mW}$ picosecond and the $20 \mathrm{~mW}$ femtosecond laser before taking another set of transient data at the same location. We controlled the illumination time to be the same for all samples.

We fitted each Stokes and anti-Stokes individually with Gaussian peak(s) with a linear baseline. For CTP and NBT, two peaks are needed to best fit the Stokes peaks, however, the corresponding anti-Stokes cannot be deconvoluted into two peaks because of the lower signal-tonoise ratio. Therefore, we added the peak area of the two fitted Stokes peaks together for following analysis. For MTP, PTP and BT, we used one Gaussian peak to fit both anti-Stokes and Stokes peaks. 
To determine the detector efficiency, we obtained the specification sheets for the gratings (600 gr/mm blazed at $750 \mathrm{~nm}$, Richardson Gratings) and the InGaAs array (Pylon-IR 1.7, Princeton Instruments). We then calculated the detector efficiency by multiplying the grating and the InGaAs array efficiency at the anti-Stokes and Stokes scattering wavelength.

To obtain the normalized anti-Stokes and Stokes scattering intensities, we divided the peak amplitude obtained from the peak fitting by the detector efficiency and extinction factor at corresponding wavelength. An example of the calculation for the $1080 \mathrm{~cm}^{-1}$ vibration mode is shown in Equation S2, S3.

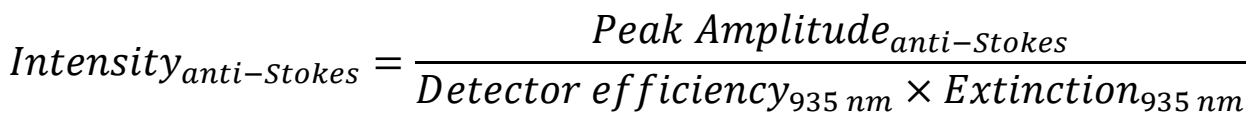

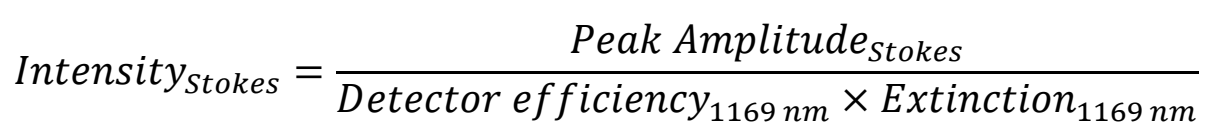

Then we calculated the excited/ground state population ratio with Equation S4

$$
\frac{\text { Excited }}{\text { Ground }} \text { Vibrational State Population Ratio }=\frac{\text { Intensity }_{\text {anti-Stokes }}}{C \times \text { Intensity }_{\text {Stokes }}}
$$

where $\mathrm{C}$ equals $\left(\frac{\omega_{\text {anti-Stokes }}}{\omega_{\text {Stokes }}}\right)^{3}$.

We propagated the errors from peak fitting to get the error for each population ratio value. We plotted the calculated population ratio as a function of delay time to get the transient kinetic traces, which are then fitted with an exponential decay convoluted with the instrument response. For each aromatic thiol, we obtained six kinetic traces at three different sample spots. We define the baseline population ratio as the baseline, the population ratio increase at time zero as transient increase, and the time constant of the exponential decay as lifetime. The we ran $t$ tests for baseline, 
transient increase and lifetime with every two aromatic thiols to determine the significance of difference.

References

(1) Frens, G. Controlled Nucleation for the Regulation of the Particle Size in Monodisperse Gold Suspensions. Nat. Phys. Sci. 1973, 241, 20-22. https://doi.org/10.1038/physci241020a0.

(2) Keller, E. L.; Frontiera, R. R. Ultrafast Nanoscale Raman Thermometry Proves Heating Is Not a Primary Mechanism for Plasmon-Driven Photocatalysis. ACS Nano 2018, 12, 58485855. https://doi.org/10.1021/acsnano.8b01809. 